\title{
Timing jitter of Raman solitons
}

\author{
Gengu Zhou, ${ }^{1,2}$ Ming XIN, ${ }^{1}$ Franz X. KAeRtner, ${ }^{1,2,3,4}$ And GuOQIng \\ $\mathrm{CHANG}^{1,3, *}$
}

\author{
${ }^{1}$ Center for Free-Electron Laser Science, DESY, Notkestraße 85, 22607 Hamburg, Germany \\ ${ }^{2}$ Physics Department, University of Hamburg, Luruper Chaussee 149, 22761 Hamburg, Germany \\ ${ }^{3}$ The Hamburg Centre for Ultrafast Imaging, Luruper Chaussee 149, 22761 Hamburg, Germany \\ ${ }^{4}$ Department of Electrical Engineering and Computer Science and Research Laboratory of Electronics, \\ Massachusetts Institute of Technology, 77 Massachusetts Avenue, Cambridge, MA 02139, USA \\ *Corresponding author: guoqing.chang@desy.de
}

Received XX Month XXXX; revised XX Month, XXXX; accepted XX Month XXXX; posted XX Month XXXX (Doc. ID XXXXX); published XX Month XXXX

\begin{abstract}
We study the relative intensity noise (RIN) and timing jitter of a Raman soliton. We demonstrate that the RIN of an excitation pulse causes center-wavelength fluctuations of the resulting Raman soliton which translates by fiber dispersion into relative timing jitter (RTJ) between the Raman soliton and the excitation pulse. The Raman soliton's absolute timing jitter is dominated by the excitation pulse's timing jitter at low frequency and by the RTJ at high frequency. The experimental study reveals that RTJ can be significantly reduced by reducing the accumulated fiber dispersion (e.g., using less dispersive fibers with shorter length) experienced by the Raman soliton. (C) 2015 Optical Society of America
\end{abstract}

OCIS codes: (190.4370) Nonlinear optics, fibers; (320.2250) Femtosecond phenomena.

http://dx.doi.org/10.1364/OL.99.099999

When a femtosecond pulse propagates inside an optical fiber with anomalous dispersion, interplay between intra-pulse Raman scattering and soliton formation results in a Raman soliton selffrequency shift [1, $\underline{2}]$; that is, the Raman soliton red shifts continuously during propagation while maintaining its transform-limited hyperbolic secant profile [3]. Capable of providing wavelength tunable femtosecond pulses at desired wavelengths that cannot be directly obtained from mode-locked ultrafast lasers, Raman soliton sources have found important applications in spectroscopy and microscopy [ $\underline{4}-\underline{6}]$. As a particular example of nonlinear wavelength conversion, difference-frequency generation (DFG) between a Raman soliton and the pulse that generates the Raman soliton has been widely used in obtaining femtosecond pulses in the mid-infrared (mid-IR) range [7-13]. Since the Raman soliton and the excitation pulse share the same repetition rate and carrier-envelope phase (CEP) offset, the resulting DFG source-if the excitation pulse's repetition rate is stabilized-becomes a mid-IR frequency comb with its CEP offset automatically set at zero. Such a mid-IR frequency comb constitutes an enabling tool for molecular spectroscopic applications because many molecules have their fingerprints in the mid-IR wavelength range.

Among many quantities that characterize a femtosecond source, timing jitter and relative intensity noise (RIN) are of particular importance and determine whether the femtosecond source is "quiet" enough for the intended applications, such as mid-IR frequency combs. Several groups have characterized the RIN or timing jitter of their Raman soliton sources [ $[\underline{7}, \underline{14}-\underline{17}]$; however a detailed theoretical and experimental study of the transfer of an excitation pulse's RIN to the resulting Raman soliton's timing jitter and how to minimize this timing jitter is absent. In this letter, we carry out a detailed study on a Raman soliton's timing jitter, and find that the relative timing jitter (RTJ) between a Raman soliton and the excitation pulse dominates at high frequency the Raman soliton's absolute timing jitter. We demonstrate that a Raman soliton's RTJ can be substantially reduced using fibers with less dispersion and shorter length.

The timing jitter and RIN of a Raman soliton source are connected by soliton formation and subsequent Raman self-frequency shift, which can be accurately modeled by the generalized nonlinear Schrödinger equation (GNLSE) taking into account dispersion, selfphase modulation, self-steepening, and Raman scattering [3] . We solve the GNLSE to simulate a 50-fs, hyperbolic-secant pulse centered at $1.035 \mu \mathrm{m}$ propagating inside a photonic crystal fiber (PCF), which is commercially available from NKT Photonics A/S. The fiber exhibits zero-dispersion at $0.945 \mu \mathrm{m}$ with a mode field diameter of $2.8 \mu \mathrm{m}$ at $1.035 \mu \mathrm{m}$, corresponding to a fiber nonlinearity of $23 \mathrm{~W}^{-1} \mathrm{~km}^{-1}$. We denote this fiber as PCF-945 in this paper. In the simulation, we fit the experimental dispersion curve provided by the manufacturer with a $12^{\text {th }}$-order polynomial (inset of Fig. 1(a)).
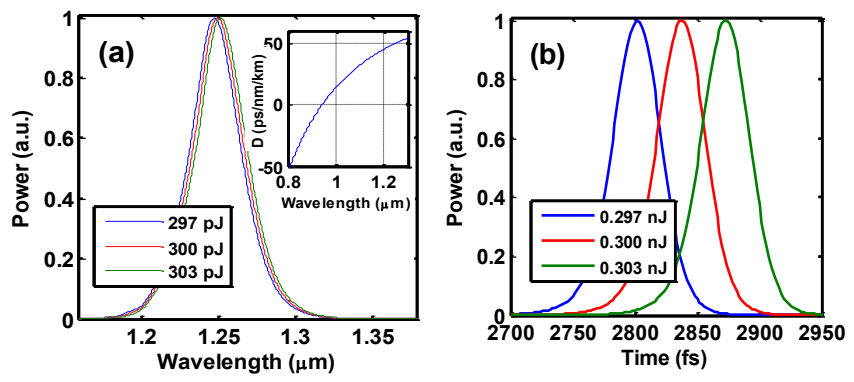

Fig 1. Simulation results for propagating a 50 -fs pulse (initial center wavelength at $1035 \mathrm{~nm}$ ) through $53-\mathrm{cm}$ PCF-945 with the zero-dispersion wavelength at $945 \mathrm{~nm}$ ). Three input pulse energies are chosen representing a $\pm 1 \%$ energy variation with respect to $300 \mathrm{pJ}$. (a) Raman soliton spectra and (b) Raman soliton pulses corresponding to different excitation pulse energies: $297 \mathrm{pJ}$ (blue), $300 \mathrm{pJ}$ (red), and $303 \mathrm{pJ}$ (green). Inset of (a) shows the dispersion curve of PCF-945. 
Figure 1(a) shows the simulated Raman soliton spectra for three different excitation pulse energies (297 pJ, 300 pJ, and 303 pJ) after propagating through 53-cm PCF-945. The corresponding three Raman solitons have a pulse energy of $178.0 \mathrm{pJ}, 179.2 \mathrm{pJ}$, and $180.4 \mathrm{pJ}$, respectively-a smaller relative energy fluctuation than the excitation pulses $(0.67 \%$ versus $1 \%)$, which implies that a Raman soliton could exhibit lower RIN than the excitation pulse. As Fig. 1(a) shows, these three Raman solitons are centered at different wavelengths (i.e., 1.248 $\mu \mathrm{m}, 1.250 \mu \mathrm{m}$, and $1.252 \mu \mathrm{m}$ at the fiber's output); therefore during Raman self-frequency shift, they propagate at different group velocities due to the fiber dispersion. Propagating in the anomalous dispersion region of the PCF, a Raman soliton with longer center wavelength travels slower than one with shorter center wavelength, and accumulates a larger temporal delay with respect to the excitation pulse at $1.035 \mu \mathrm{m}$. Figure 1(b) plots the three Raman soliton pulses corresponding to the excitation pulse energy of $297 \mathrm{pJ}$ (blue), $300 \mathrm{pJ}$ (right), and 303 pJ (green) in the time domain; they peak at $2802 \mathrm{fs,}$ $2838 \mathrm{fs}$, and $2874 \mathrm{fs}$, respectively. Note that we solve the GNLSE using a frame of reference moving with the group velocity at $1.035 \mu \mathrm{m}$ defined by the PCF's dispersion. The temporal peaking position of a Raman soliton therefore indicates the relative delay experienced by the Raman soliton with respect to a virtual pulse linearly propagating through the fiber with the center wavelength at $1.035 \mu \mathrm{m}$.

The results in Fig. 1(b) show that 1\% change in the excitation pulse energy leads to 36 -fs change of temporal delay. It suggests that the excitation pulse's RIN will be converted into RTJ between the Raman soliton and the excitation pulse. This RTJ differs from the Raman soliton's absolute timing jitter with respect to the lab, which should include contribution from the excitation pulse's timing jitter. However, RTJ is of particular importance for nonlinear wavelength conversion that involves both spatially and temporally overlapping a Raman soliton and its excitation pulse in a nonlinear crystal. For example, DFG between these two pulses will transfer their RTJ to the derived mid-IR comb source manifesting as broadened comb lines and increased RIN. Minimizing RTJ of a Raman soliton is crucial for realizing a low-noise mid-IR frequency comb desired by molecular precision spectroscopy.

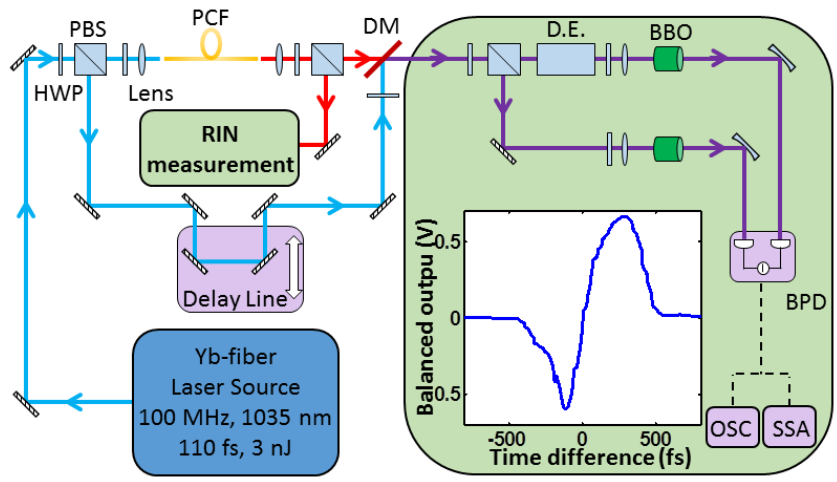

Fig. 2. Schematic setup to characterize RIN and RTJ of Raman soliton source. PBS: polarization beam splitter, PCF: photonic crystal fiber, HWP: Half-wave plate, DM: dichroic mirror, D.E.: delay element, BBO: beta- $\mathrm{BaB}_{2} \mathrm{O}_{4}$ crystal, BPD: balanced photodetector, OSC: oscilloscope, SSA: signal source analyzer.

Raman soliton centered at a desired wavelength can be achieved by different combinations of fiber dispersion, fiber length, and excitation pulse energy and pulse duration. Guided by simulation, we carry out a detailed experimental study on how to optimize such a parameter combination. Figure 2 illustrates the experimental setup. The Raman soliton source is derived from a home-built $\mathrm{Yb}$-fiber laser system including a 100-MHz Yb-fiber oscillator centered at $1035 \mathrm{~nm}$, an Ybfiber amplifier, and a pulse compressor. The Yb-fiber oscillator operates in the stretched-pulse mode-locking regime enabled by nonlinear polarization evolution. We optimize both the mode-locking state and net cavity-dispersion to minimize the oscillator's RIN, which is shown as the green curve in Fig. 3. The oscillator exhibits an integrated RIN of $0.018 \%$ from $10 \mathrm{~Hz}$ to $10 \mathrm{MHz}$. The Yb-fiber amplifier is optimized as well such that it only slightly degrades the RIN of the amplified pulses (red curve in Fig. 3). The measured RIN in the frequency range of $10 \mathrm{~Hz}$ to $2 \mathrm{kHz}$ is buried under the instrument noise floor represented by the blue curve.

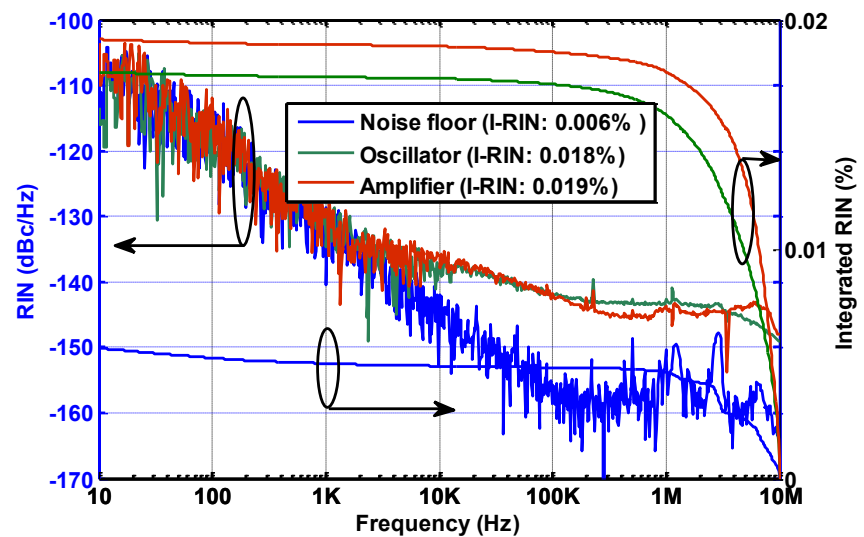

Fig. 3. RIN spectra of oscillator (green curve) and amplifier (red curve). Instrument noise floor is shown as blue curve. The corresponding integrated RIN curves are obtained by integrating the RIN spectra from $10 \mathrm{MHz}$ to $10 \mathrm{~Hz}$.

The laser system provides 3-nJ, 110-fs pulses, which are then split into two arms using a half-wave plate and a polarization beam splitter (PBS). By rotating the half-wave plate, we can continuously vary the optical power coupled into the PCF, and hence obtain a Raman soliton at a desired wavelength. At the fiber output, a dichroic mirror (DM) is used to spatially combine the Raman soliton pulse and the reference pulse before sending them into a balanced optical cross-correlator (BOC) [18] to measure their RTJ. More specific, the power of the input pulse pair is first separated into two branches by a PBS. In each branch, the pulse pair is focused into a 10-mm Type-I phase-matched beta$\mathrm{BaB}_{2} \mathrm{O}_{4}$ (BBO) crystal (cutting angle: 21.9') for sum-frequency generation (SFG). A delay element (DE) that consists of two half-inch cubes made of N-SF1 glass is inserted into one branch to provide a 150 -fs delay offset. The two SFG signals are then filtered out by band pass filters (centered at $555 \mathrm{~nm}$, bandwidth $40 \mathrm{~nm}$ ) and finally detected by a balanced photodetector (BPD). The BOC curve-the BPD output voltages with respect to the time delay of the two input pulses at the DM-is measured and shown as the inset of Fig. 2. Apparently, BOC converts the timing fluctuations between the two pulses to voltage fluctuations at BPD output. In each BPD channel, the RIN of the Raman soliton or the excitation pulse also contributes to the voltage change, which as the common-mode noise is cancelled out in the balance detection. A delay line in the reference arm before the DM guarantees that the timing fluctuations are within the detection range of the BOC. BOCs have been widely used in ultra-low timing jitter characterization $[\underline{19} \underline{20}]$, timing error detection in timing distribution systems $[\underline{21}, \underline{22}]$, and pulse synthesis from independent mode-locked lasers [23].

Figure 4(a) plots the RIN of Raman solitons centered at $1200 \mathrm{~nm}$ (red curve) and $1300 \mathrm{~nm}$ (blue curve) generated from 205-cm PCF945. Comparison between Fig. 4(a) and Fig. 3 shows that a Raman soliton's RIN and the excitation pulse's RIN share similar frequency dependency in the frequency range between $1 \mathrm{kHz}$ and $10 \mathrm{MHz}$. The spiky structures in the Raman soliton's RIN in the range between 100 $\mathrm{Hz}$ and $1 \mathrm{kHz}$ might be also caused by the excitation pulse's RIN, which is buried under the instrument noise floor in this frequency range. Additionally, ambient acoustic noise may contribute as well.

Figure 4(b) presents the measured RTJ for these two Raman solitons. Clearly these RTJ curves follow exactly the same frequency dependency as the Raman soliton's RIN curves in Fig. 4(a), implying that the RTJ is mainly caused by the excitation pulse's RIN. Note that the RTJ differs from the excitation pulse's timing jitter, which is determined by the mode-locked Yb-fiber oscillator and is ultimately limited by quantum noise. For a free-running Yb-fiber oscillator operating in the stretched-pulse mode-locking regime, the main 
quantum noise arises from the amplified spontaneous emission, which adds to the intra-cavity circulating pulse when it propagates through the Yb-fiber. Consequently, the temporal center-of-gravity of the circulating pulse undergoes a random walk, leading to a timing jitter with a power spectral density proportional to $f^{-2}$ [24]. In contrast, the RTJ between a Raman soliton and the excitation pulse begins to flatten out beyond the Yb-fiber's relaxation oscillation frequency $(\sim 1 \mathrm{kHz})$, closely following the tendency of the excitation pulse's RIN. Since a mode-locked laser's RIN remains finite at any frequency, the resulting center-wavelength fluctuation of a derived Raman soliton is limited within a certain range, which in turn results in a bounded RTJ via fiber dispersion.

Unbounded at zero frequency, the timing jitter of a free-running $\mathrm{Yb}$ fiber oscillator is dominated by low-frequency jitter. For example, the experimental results in Ref. [19] show that, for an Yb-fiber oscillator mode-locked in the stretched-pulse regime, the contribution of highfrequency timing jitter integrated from $10 \mathrm{kHz}$ to $40 \mathrm{MHz}$ is only $\sim 0.2$ fs. As a comparison, the main contribution in a Raman soliton's RTJ concentrates at from high frequency. The two curves corresponding to the RMS timing jitter in Fig. 4(b) clearly indicate that the jitter contribution below $1 \mathrm{MHz}$ is negligible. A Raman soliton's absolute timing jitter includes both the excitation pulse's timing jitter and the RTJ between these two pulses. For a Raman soliton excited by pulses produced from a free-running oscillator, its timing jitter is dominated by the excitation pulse's timing jitter at low frequency, exhibiting $f^{-2}$ character; RTJ contributes mainly at high frequency.
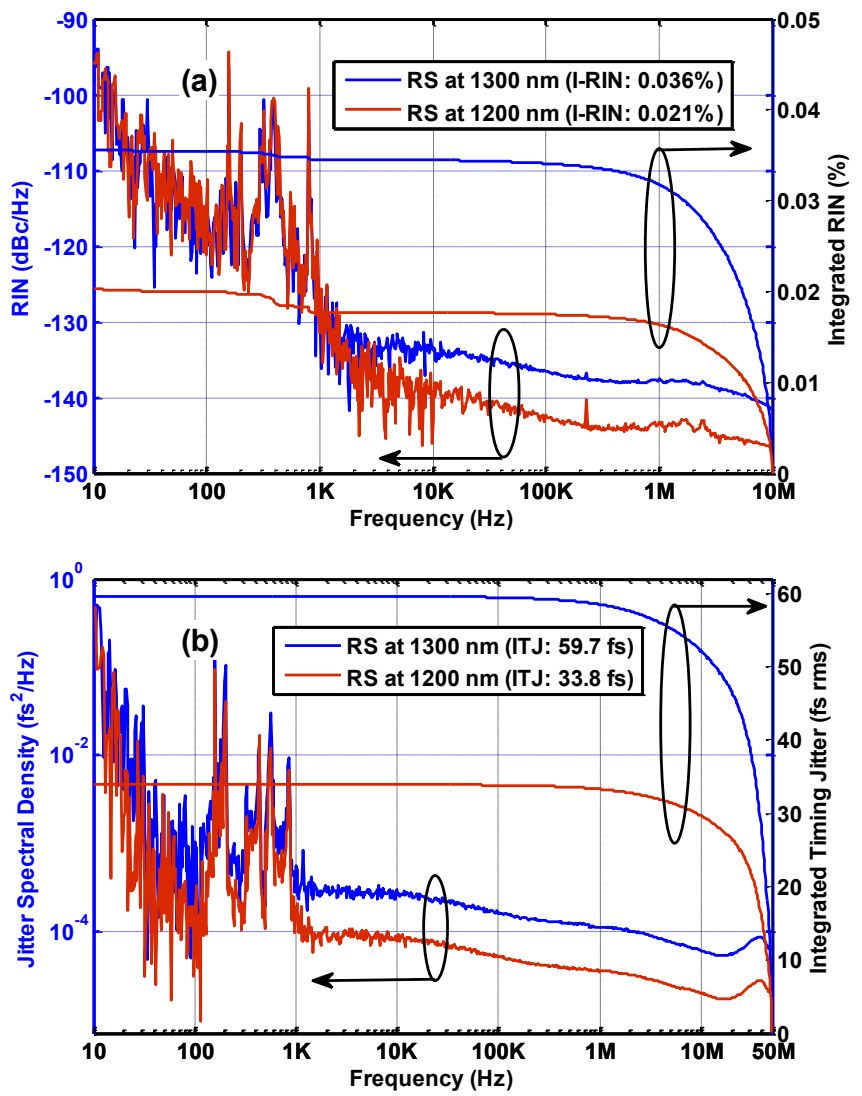

Fig. 4. (a) RIN measurement of Raman solitons at $1200 \mathrm{~nm}$ (red curve) and 1300 $\mathrm{nm}$ (blue curve). (b) Relative timing jitter of Raman solitons at $1200 \mathrm{~nm}$ (red curve) and $1300 \mathrm{~nm}$ (blue curve). These two Raman solitons are generated from 205-cm PCF-945. RS: Raman soliton, I-RIN: integrated RIN, ITJ: integrated timing jitter.

As Fig. 4(b) shows, the integrated RTJs from $50 \mathrm{MHz}$ to $10 \mathrm{~Hz}$ are $33.8 \mathrm{fs}$ and $59.7 \mathrm{fs}$, which are comparable to the pulse duration $(\sim 100$ fs) of the two Raman solitons at $1200 \mathrm{~nm}$ and $1300 \mathrm{~nm}$. If a Raman soliton and the excitation pulse are employed in nonlinear wavelength mixing (e.g., DFG, SFG, four-wave mixing etc.), such a huge RTJ will be converted into large RIN and timing jitter of the newly derived pulse. For example, we measure the RIN of the SFG signal at the output of one of the two BBO crystals. This RIN spectrum resembles the RT] spectrum, and the integrated RIN is $\sim 10 \%$, about two orders of magnitude higher than the RIN of the Raman soliton pulse and the excitation pulse.
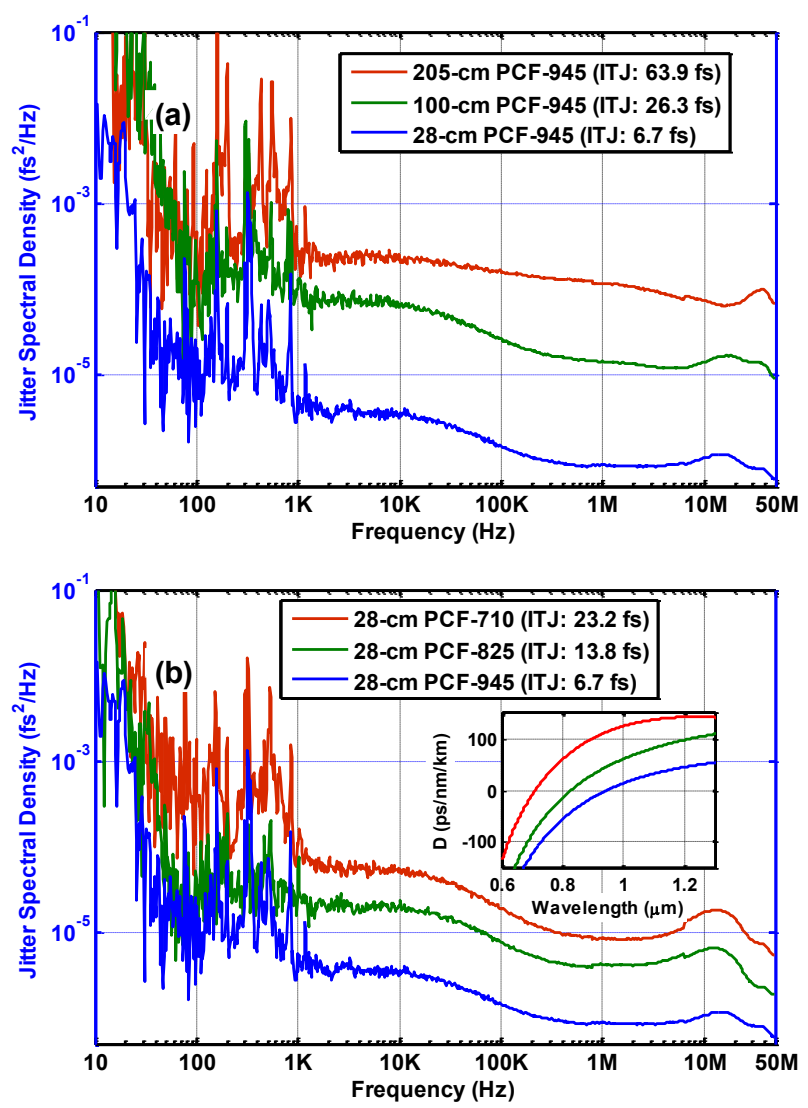

Fig. 5. Timing jitter spectra of the 1250-nm Raman soliton generated by (a) PCF945 with different fiber lengths: $28 \mathrm{~cm}$ (blue curve), $100 \mathrm{~cm}$ (green curve), and $205 \mathrm{~cm}$ (red curve), and by (b) 28-cm fibers with the zero-dispersion wavelength at $710 \mathrm{~nm}$ (red curve), $825 \mathrm{~nm}$ (green curve), and $945 \mathrm{~nm}$ (blue curve). IT): integrated timing jitter. Inset of (b) shows the dispersion curves of these three fibers: PCF-710 (red), PCF-825 (green), and PCF-945 (blue).

Reducing timing jitter of a pulse train can be achieved by a feedback loop. For example, locking a fiber oscillator's repetition rate to a microwave reference using a feedback loop can significantly suppress the low-frequency timing jitter, which constitutes the main contribution to a free-running oscillator's timing jitter. The bandwidth limitation $(<1 \mathrm{MHz}$ ) renders a feedback loop incapable of removing the high-frequency (1-50 MHz) components in the RTJ between a Raman soliton and its excitation pulse. We have to rely on optimizing the system parameters to efficiently reduce a Raman soliton's RTJ. We first investigate the effect of fiber length. Using shorter fiber length with higher excitation pulse energy can generate Raman solitons at the same wavelength, but with different RTJs. Figure 5(a) plots the RTJ spectra of Raman solitons centered at $1250 \mathrm{~nm}$ generated by fiber PCF-945 with different fiber lengths: $28 \mathrm{~cm}$ (blue curve), $100 \mathrm{~cm}$ (green curve), and $205 \mathrm{~cm}$ (red curve); the corresponding integrated RTJs are 6.7 fs, 26.3 fs, and 63.9 fs, respectively. The Raman soliton generated by 28-cm PCF-945 shows the lowest RTJ. These experimental results are expected because, for the same amount of wavelength shift, a Raman soliton experiences less dispersion effect in a shorter PCF and therefore accumulates less timing jitter.

Raman solitons at a desired wavelength can also be obtained using fibers with different dispersions. Besides PCF-945, we choose two more commercially available PCFs-PCF-825 and PCF-710 with their zero-dispersion wavelength at $825 \mathrm{~nm}$ and $710 \mathrm{~nm}$-for a 
comparison. We fix the fiber length at $28 \mathrm{~cm}$ and adjust the excitation pulse energy to tune the center wavelength of a Raman soliton. Figure 5(b) shows the RTJ spectra of the $1250 \mathrm{~nm}$ Raman solitons generated by these three fibers. Their dispersion curves (inset of Fig. 5(b)) show that, in our interested wavelength range of 1035-1300 nm, PCF-945 has the least dispersion and PCF-710 has the largest dispersion. As we expect, the Raman soliton generated from 28-cm PCF-945 exhibits the least RT) (blue curve in Fig. 5(b)) while PCF-710 generates the Raman soliton with largest RTJ (red curve in Fig. 5(b)).

To further investigate the RTJ dependence on fiber length and dispersion, we measure the RTJ of Raman solitons centered at 1200 $\mathrm{nm}, 1225 \mathrm{~nm}, 1275 \mathrm{~nm}$, and $1300 \mathrm{~nm}$ as well. Figure 6(a) summarizes the integrated RTJ of these Raman solitons generated by PCF-945 at different fiber lengths, demonstrating that shorter fiber length leads to less RTJ at all Raman soliton wavelengths. Figure 6(b) presents the integrated RT]s corresponding to different PCFs all at the same fiber length of $28 \mathrm{~cm}$, showing that fiber with less dispersion results in less RTJ for Raman solitons centered at the same wavelength.
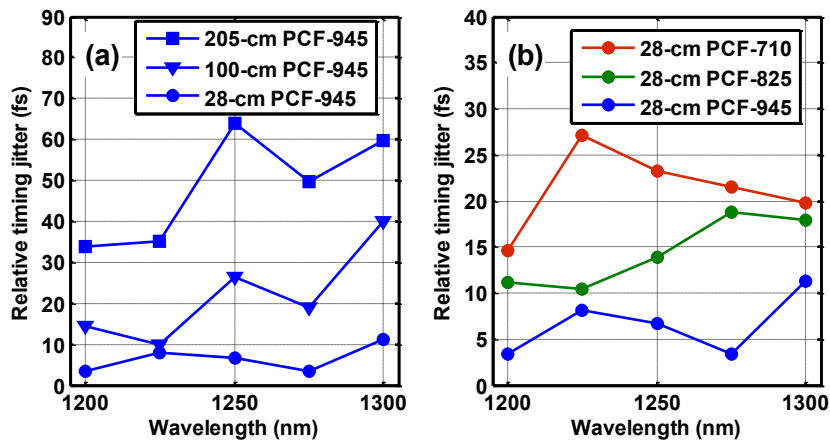

Fig. 6. Integrated RTJ of Raman solitons generated by (a) PCF-945 with different fiber lengths: $28 \mathrm{~cm}$ (blue circles), 100 -cm PCF-945 (blue triangles), 205-cm PCF945 (blue squares) and by (b) 28-cm of PCF-945 (blue circles), PCF-825 (green circles), and PCF-710 (red circles).

The results presented in Fig. 6 suggest that substantial reduction of RTJ of a Raman soliton can be achieved by minimizing the accumulated dispersion in a PCF. In practice, however, we have to keep the accumulated dispersion beyond a certain value. For an excitation pulse at a given duration, shifting a Raman soliton to a desired wavelength using less dispersive fiber with shorter length necessitates increasing the excitation pulse energy. With enough pulse energy, the excitation pulse forms a higher-order soliton at the beginning and then breaks into $\mathrm{N}$ fundamental solitons mainly caused by higher-order dispersion. These $\mathrm{N}$ fundamental solitons experience different amount of Raman self-frequency shift, leading to multiple Raman solitons appearing at different center wavelengths. If the accumulated dispersion is too small, these N Raman solitons spectrally merge together manifesting as a supercontinuum. Consequently access to a specific Raman soliton using an optical bandpass filter becomes impractical. Furthermore, increased excitation pulse energy causes stronger nonlinearity during soliton fission, which is more sensitive to the excitation pulse's RIN. As a result, a resulting Raman soliton may suffer from higher RIN, which offsets the benefit of reducing the accumulated dispersion. Indeed, we have measured the RIN and RTJ of Raman solitons generated from 18cm PCF-945; these Raman solitons have RTJs 2 times larger than their counterparts obtained from 28-cm PCF-945 due to the increased RIN. The limitation imposed by increased excitation-pulse energy can be mitigated using shorter excitation pulses. For fixed excitation-pulse energy, short pulse duration leads to a soliton with smaller order (i.e., smaller N); the resulting Raman solitons also have shorter duration and red shift with a much faster rate. For example, for a 10-fs excitation pulse centered at $800 \mathrm{~nm}$ with $325 \mathrm{pJ}$ pulse energy, 15-cm PCF-710 causes $>300 \mathrm{~nm}$ wavelength shift, generating a spectrally well-isolated Raman soliton at $1120 \mathrm{~nm}$ [25].
In conclusion, we demonstrate that the RIN of an excitation pulse causes center-wavelength fluctuations of the resulting Raman soliton and then translates into the RTJ between the Raman soliton and the excitation pulse by fiber dispersion. A Raman soliton's absolute timing jitter is dominated at low frequency by the excitation pulse's timing jitter and at high frequency by the RTJ. Our experimental results suggest that RTJ can be significantly reduced by minimizing the accumulated fiber dispersion experienced by the Raman soliton using fibers with less dispersion and shorter length. Ongoing work is to further optimize our laser system (e.g., shorten the excitation pulse duration) to achieve a Raman soliton with attosecond-level RTJ, which is crucial for implementing a low noise mid-IR frequency comb via DFG between the Raman soliton and the excitation pulse.

Funding. Helmholtz Association through Helmholtz Young Investigator Group (VH-NG-804); Helmholtz-CAS Joint Research Group (HCJRG 201).

Acknowledgment. The authors acknowledge financial support by the excellence cluster "The Hamburg Centre for Ultrafast Imaging Structure, Dynamics and Control of Matter at the Atomic scale" of the Deutsche Forschungsgemeinschaft.

\section{References}

1. F. Mitschke and L. Mollenauer, Opt. Lett. 11, 659 (1986).

2. J. Gordon, Opt. Lett. 11, 662 (1986).

3. G. P. Agrawal, Nonlinear Fiber Optics (Academic, 2013).

4. J. W. Walewski, M. R. Borden, S. T. Sanders, Appl. Phys. B. 79, 937 (2004).

5. A. A. Ivanov, A. A. Podshivalov, and A. M. Zheltikov, Opt. Lett. 31, 3318 (2006).

6. N. G. Horton, K. Wang, D. Kobat, C. G. Clark, F. W. Wise, C. B. Schaffer, and C. Xu, Nat. Photon. 7, 205 (2013).

7. D. Winters, P. Schlup, and R. Bartels, Opt. Lett. 35, 2179 (2010).

8. T. W. Neely, T. A. Johnson, and S. A. Diddams, Opt. Lett. 36, 4020 (2011).

9. A. Ruehl, A. Gambetta, I. Hartel, M. E. Fermann, K. S. E. Eikema, and M. Marangoni, Opt. Lett. 37, 2232 (2012).

10. C. R. Phillips, J. Jiang, C. Mohr, C. Langrock, M. Snure, D. Bliss, M. Zhu, I. Hartl, J. S. Harris, M. E. Fermann, and M. M. Fejer, Opt. Lett. 37, 2928 (2012).

11. A. Gambetta, N. Coluccelli, M. Cassinerio, D. Gatti, P. Laporta, G. Galzerano, and M. Marangoni, Opt. Lett. 38, 1155 (2013).

12. N. Coluccelli, M. Cassinerio, A. Gambetta, P. Laporta, and G. Galzerano, Opt. Lett. 39, 1661 (2014).

13. D. Sanchez, M. Hemmer, M. Baudisch, K. Zawilski, P. Schunemann, H. Hoogland, R. Holzwarth, and J. Biegert, Opt. Lett. 39, 6883 (2014).

14. J. Chen, F. O. Ilday, and F. X. Kärtner, paper TuB9, in Advanced Solid State Photonics (OSA, 2006).

15. D. G. Winters, P. Schlup, and R. A. Bartels, paper ATuA6, in Advanced Solid State Photonics (OSA, 2011).

16. C. H. Hage, B. Kibler, E. R. Andresen, S. Michel, H. Rigneault, A. Courjaud, E. Mottay, J. M. Dudley, G. Millot, and C. Finot, Proc. SPIE 8071, Nonlinear Optics and Applications V, $80710 \mathrm{I}$ (2011).

17. J. Lim, H. -W. Chen, S. H. Xu, Z. M. Yang, G. Q. Chang, and F. X. Kärtner, Opt. Lett. 39, 2060 (2014).

18. T. R. Schibli, J. Kim, O. Kuzucu, J. Gopinath, S. N. Tandon, G. S. Petrich, L. A. Kolodziejski, J. G. Fujimoto, E. P. Ippen, and F. X. Kärtner, Opt. Lett. 28, 947 (2003).

19. Y. Song, K. Jung, and J. Kim, Opt. Lett. 36, 1761 (2011).

20. A. J. Benedick, J. G. Fujimoto, and F. X. Kärtner, Nat. Photon. 6, 97 (2012).

21. J. Kim, J. A. Cox, J. Chen, and F. X. Kärtner, Nat. Photonics 2, 733 (2008).

22. M. Xin, K. Safak, M. Y. Peng, P. T. Callahan, and F. X. Kärtner, Opt. Express 22, 14904 (2014).

23. J. A. Cox, W. P. Putnam, A. Sell, A. Leitenstorfer, and F. X. Kärtner, Opt. Lett. 37, 3579-3581 (2012).

24. H. A. Haus and A. Mecozzi, IEEE J. Quantum Electron. 29, 983 (1993).

25. G. Q. Chang, L. -J. Chen, and F. X. Kärtner, Opt. Lett. 35, 2361 (2010). 


\section{Full References List}

1. F. M. Mitschke and L. F. Mollenauer, "Discovery of the soliton selffrequency shift," Opt. Lett. 11, 659-661 (1986)

2. J. P. Gordon, "Theory of the soliton self-frequency shift," Opt. Lett. 11, 662664 (1986)

3. G. P. Agrawal, Nonlinear Fiber Optics (Academic, 2013).

4. J. W. Walewski, M. R. Borden, S. T. Sanders, "Wavelength-agile laser system based on soliton self-shift and its application for broadband spectroscopy," Appl. Phys. B. 79, 937 (2004).

5. A. A. Ivanov, A. A. Podshivalov, and A. M. Zheltikov, "Frequency-shifted megawatt soliton output of a hollow photonic-crystal fiber for timeresolved coherent anti-Stokes Raman scattering microspectroscopy," Opt. Lett. 31, 3318-3320 (2006).

6. Nicholas G. Horton, Ke Wang, Demirhan Kobat, Catharine G. Clark, Frank W. Wise, Chris B. Schaffer \& Chris Xu, "In vivo three-photon microscopy of subcortical structures within an intact mouse brain," Nat. Photon. 7, 205 (2013).

7. David G. Winters, Philip Schlup, and Randy A. Bartels, "Subpicosecond fiber-based soliton-tuned mid-infrared source in the 9.7-14.9 $\mu \mathrm{m}$ wavelength region," Opt. Lett. 35, 2179-2181 (2010).

8. Tyler W. Neely, Todd A. Johnson, and Scott A. Diddams, "High-power broadband laser source tunable from $3.0 \mu \mathrm{m}$ to $4.4 \mu \mathrm{mbased}$ on a femtosecond Yb:fiber oscillator," Opt. Lett. 36, 4020-4022 (2011).

9. Axel Ruehl, Alessio Gambetta, Ingmar Hartl, Martin E. Fermann, Kjeld S. E. Eikema, and Marco Marangoni, "Widely-tunable mid-infrared frequency comb source based on difference frequency generation," Opt. Lett. 37, 2232-2234 (2012).

10. C. R. Phillips, J. Jiang, C. Mohr, C. Langrock, M. Snure, D. Bliss, M. Zhu, I. Hartl, J. S. Harris, M. E. Fermann, and M. M. Fejer, "Widely tunable midinfrared difference frequency generation in orientation-patterned GaAs pumped with a femtosecond Tm-fiber system," Opt. Lett. 37, 2928 (2012).

11. Alessio Gambetta, Nicola Coluccelli, Marco Cassinerio, Davide Gatti, Paolo Laporta, Gianluca Galzerano, and Marco Marangoni, "Milliwatt-level frequency combs in the 8-14 $\mu \mathrm{m}$ range via difference frequency generation from an Er:fiber oscillator," Opt. Lett. 38, 1155-1157 (2013).

12. Nicola Coluccelli, Marco Cassinerio, Alessio Gambetta, Paolo Laporta, and Gianluca Galzerano, "High-power frequency comb in the range of 2$2.15 \mu \mathrm{m}$ based on a holmium fiber amplifier seeded by wavelengthshifted Raman solitons from an erbium-fiber laser," Opt. Lett. 39, 16611664 (2014)

13. D. Sánchez, M. Hemmer, M. Baudisch, K. Zawilski, P. Schunemann, H. Hoogland, R. Holzwarth, and J. Biegert, "Broadband mid-IR frequency comb with $\mathrm{CdSiP}_{2}$ and $\mathrm{AgGaS}_{2}$ from an Er,Tm:Ho fiber laser," Opt. Lett. 39, 6883-6886 (2014).

14. J. Chen, F. O. Ilday, and F. X. Kaertner, "Soliton Self-Frequency Shift from $1.03 \mu \mathrm{m}$ to $1.55 \mu \mathrm{m}$," in Advanced Solid-State Photonics, Technical Digest (Optical Society of America, 2006), paper TuB9.

15. D. Winters, P. Schlup, and R. Bartels, "Generation and Stability Characterization of Fiber-Based Difference Frequency Generation Tuned Through Controlled Soliton Self-Frequency-Shifting," in Advances in Optical Materials, OSA Technical Digest (CD) (Optical Society of America, 2011), paper ATuA6.

16. C. H. Hage, B. Kibler, E. R. Andresen, S. Michel, H. Rigneault, A. Courjaud, E. Mottay, J. M. Dudley, G. Millot, and C. Finot, “Optimization and characterization of a femtosecond tunable light source based on the soliton self-frequency shift in photonic crystal fiber," Proc. SPIE 8071, Nonlinear Optics and Applications V, 807101 (2011).

17. Jinkang Lim, Hung-Wen Chen, Shanhui Xu, Zhongmin Yang, Guoqing Chang, and Franz X. Kärtner, " $3 \mathrm{GHz}$, watt-level femtosecond Raman soliton source," Opt. Lett. 39, 2060-2063 (2014).

18. T. R. Schibli, J. Kim, O. Kuzucu, J. T. Gopinath, S. N. Tandon, G. S. Petrich, L. A. Kolodziejski, J. G. Fujimoto, E. P. Ippen, and F. X. Kaertner, "Attosecond active synchronization of passively mode-locked lasers by balanced cross correlation," Opt. Lett. 28, 947-949 (2003).

19. Youjian Song, Kwangyun Jung, and Jungwon Kim, "Impact of pulse dynamics on timing jitter in mode-locked fiber lasers," Opt. Lett. 36, 17611763 (2011).
20. Andrew J. Benedick, James G. Fujimoto \& Franz X. Kärtner, "Optical flywheels with attosecond jitter," Nat. Photon. 6, 97 (2012).

21. Jungwon Kim, Jonathan A. Cox, Jian Chen \& Franz X. Kärtner, "Drift-free femtosecond timing synchronization of remote optical and microwave sources," Nat. Photonics 2, 733 (2008).

22. Ming Xin, Kemal Şafak, Michael Y. Peng, Patrick T. Callahan, and Franz X. Kärtner, "One-femtosecond, long-term stable remote laser synchronization over a 3.5-km fiber link," Opt. Express 22, 14904-14912 (2014).

23. J. A. Cox, W. P. Putnam, A. Sell, A. Leitenstorfer, and F. X. Kärtner, "Pulse synthesis in the single-cycle regime from independent mode-locked lasers using attosecond-precision feedback," Opt. Lett. 37, 3579-3581 (2012).

24. H. A. Haus and A. Mecozzi, "Noise of mode-locked lasers," IEEE J. Quantum Electron. 29, 983 (1993).

25. Guoqing Chang, Li-Jin Chen, and Franz X. Kärtner, "Highly efficient Cherenkov radiation in photonic crystal fibers for broadband visible wavelength generation," Opt. Lett. 35, 2361-2363 (2010). 